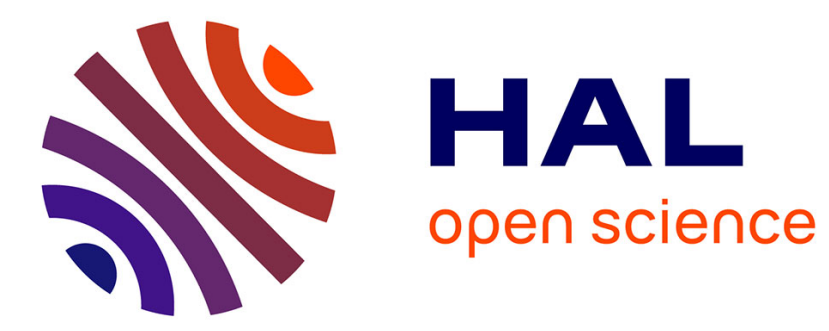

\title{
Eigenvalues in the large sieve inequality, II
}

Olivier Ramaré

\section{To cite this version:}

Olivier Ramaré. Eigenvalues in the large sieve inequality, II. Journal de Théorie des Nombres de Bordeaux, 2010, 22 (1), pp.181-196. 10.5802/jtnb.710 . hal-02572752

\section{HAL Id: hal-02572752 \\ https://hal.science/hal-02572752}

Submitted on 13 May 2020

HAL is a multi-disciplinary open access archive for the deposit and dissemination of scientific research documents, whether they are published or not. The documents may come from teaching and research institutions in France or abroad, or from public or private research centers.
L'archive ouverte pluridisciplinaire HAL, est destinée au dépôt et à la diffusion de documents scientifiques de niveau recherche, publiés ou non, émanant des établissements d'enseignement et de recherche français ou étrangers, des laboratoires publics ou privés. 


\title{
Eigenvalues in the large sieve inequality, II
}

\author{
par OLIVIER RAMARÉ
}

RÉsumÉ. Nous explorons numériquement les valeurs de la forme hermitienne

$$
\sum_{q \leq Q} \sum_{\bmod *_{q}}\left|\sum_{n \leq N} \varphi_{n} e(n a / q)\right|^{2}
$$

lorsque $N=\sum_{q \leq Q} \phi(q)$. Nous améliorons la majoration actuelle et exhibons un graphique conjectural de la distribution asymptotique de ses valeurs propres en exploitant des résultats de calculs intensifs. L'une des conséquences est que la distribution asymptotique existe probablement mais n'est pas absolument continue par rapport à la mesure de Lebesgue.

Abstract. We explore numerically the eigenvalues of the hermitian form

$$
\sum_{q \leq Q} \sum_{\bmod * q}\left|\sum_{n \leq N} \varphi_{n} e(n a / q)\right|^{2}
$$

when $N=\sum_{q \leq Q} \phi(q)$. We improve on the existing upper bound, and produce a (conjectural) plot of the asymptotic distribution of its eigenvalues by exploiting fairly extensive computations. The main outcome is that this asymptotic density most probably exists but is not continuous with respect to the Lebesgue measure.

\section{Introduction}

In [9], we explored numerically as well as theoretically the distribution of the eigenvalues of the hermitian form

$$
\sum_{q \leq Q} \sum_{\bmod { }^{*} q}\left|\sum_{n \leq N} \varphi_{n} e(n a / q)\right|^{2}
$$

when $Q^{2}$ is close to $N$. Bounds for this hermitian form are of utmost importance for arithmetical uses and are the core of what is loosely refered to as "the large sieve" and we refer the reader to [7] and [1] for more details on this subject. Let us assume that

$$
N=\Phi(Q)=\sum_{q \leq Q} \phi(q)
$$

AMS Classification : 11L03, 11L07, 11L26, 30E05 secondary : 41A10, 41A17

Keywords : Large sieve inequality, circle method, Jackson polynomials, Hausdorff moment problem 
and let us denote by $\left(\lambda_{i}\right)_{1 \leq i \leq N}$ be its eigenvalues. We produced graphics in [9] showing that the sequence of functions of $\lambda$ defined by

$$
\mathscr{D}(N, Q, \lambda)=\#\left\{i / \lambda_{i} \leq \lambda N\right\} / N
$$

seems to converge. The question has been asked as to the shape of the density involved and whether one could produce a graphical candidate for this density. The underlying idea is also that one may hope to guess what this density should be from its shape. Note that the large sieve inequality tells us that these eigenvalues are not more than $N+Q^{2}$, so that $\lambda$ is constrained to lie in $[0, L]$, with practically $L=3.5$. See also section 2 on this paper for the upper bound $L \leq 3.548$ and section 3 for a hint as to what the actual value can be.

Producing such a diagram is however more difficult than its seems. Indeed, assuming this density to be continuous, we can approximate it by the number of eigenvalues (renormalized by being divided by $N$ ) lying in a given interval. However, when this interval is too small, this number will only be 0 or 1 . We thus should guess what is the proper length to be used. It is tempting to select intervals of length about $1 / \sqrt{N}$, but $N$ being between 100 and 500, this means having only 10 to 20 intervals at our disposal. This also means computing all the eigenvalues while only using very partial information concerning them.

We decided to follow a more direct approach: since the computation of the eigenvalues in fact relied on computing powers of our matrix, say $M$, in order to build its characteristic polynomial, we set to compute the trace of these powers but only to a limited power $h \leq H=100$. This also means that we have been able to increase noticeably the size of our matrices: the largest $Q$ for which we have computed all the eigenvalues is $Q=40$, and this means 490 eigenvalues, while we computed the 100th first moments for $Q=70$, using a precision of 110 digits. This means handling matrices of size $1494 \times 1494$. These computations are somewhat more detailed in section 3. We then try to guess the form of the density. We have thus access to the quantities

$$
\int_{0}^{L} t^{h} d D(t) \quad(0 \leq h \leq H)
$$

and we aim at recovering $\int_{I} d D(t) /|I|$ for intervals $I$ as small as possible. We approximate in fact $d D(x) / d x$ by

$$
\int_{0}^{L} P(x, t) d D(t) / \int_{0}^{L} P(x, t) d t
$$

where $P(x, t)$ is a polynomial of degree $\leq n$ and which has a sharp peak at $x$. We take $n=H$. Deciding of which polynomials to take is again 
a problem. We opted for a solution involving Jackson polynomials (recalled thereafter). A similar problem, but with fairly smooth densities, has already been considered in [3] where the author resorts to Bernstein polynomials. Jackson polynomials provide a better polynomial approximation, in particular with densities that have a low level of regularity. These polynomials have the advantage of being non-negative, while having a sharp peak at fairly well-spaced points (this is false close to the edge of the range, but our distribution is very small there). To produce a diagram, we proceeded as follows

(1) we took the $n / 2$ points $x_{k}=2 k L / n$ for $k$ from 0 to $n / 2$.

(2) The points $x_{k}^{*}=2\left(x_{k} / L\right)-1$ are now well-spaced over $[-1,1]$.

(3) For each $k$, select $\ell$ such that

$$
\cos \frac{2 \pi \ell}{n+1} \geq x_{k}^{*}>\cos \frac{2 \pi(\ell+1)}{n+1}
$$

and take a linear interpolation of the relevant Jackson polynomials:

$$
P^{*}\left(x_{k}^{*}, t\right)=\lambda \tilde{K}_{\ell, n}(t)+(1-\lambda) \tilde{K}_{\ell+1, n}(t)
$$

where

$$
\lambda=\frac{x_{k}^{*}-\cos \frac{2 \pi(\ell+1)}{n+1}}{\cos \frac{2 \pi \ell}{n+1}-\cos \frac{2 \pi(\ell+1)}{n+1}} .
$$

(4) We finally choose $P\left(x_{k}, t\right)=P^{*}\left(x_{k}^{*}, 2 t-1\right)$.

Since we have all these moments at our disposal, we decided to investigate them as well. The reader will see in section 3 that they behave very regularly. We thus decided to produce a numerical fit. If a distribution can be written as $d D(t)=\delta(t) d t$ with a smooth enough function $\delta$, then these moments should behave like $A+B(\log h) / h+\mathcal{O}\left(\left(\log ^{2} h\right) / h^{2}\right)$. This is not what we obtained, see section 3 for more details.

\section{On the largest eigenvalue when $N=\Phi(Q)$}

In his lectures on sieve [10], Selberg announced that the largest eigenvalue of $(1.1)$ is indeed $\leq(1-\epsilon)\left(N+Q^{2}\right)$ for some $\epsilon>0$ and when $N=\Phi(Q)$, see (1.2). However, he provided no numerical values for $\epsilon$ and this is the aim of this section. We recall the path proposed by Selberg. Let $F_{1}$ be a smooth function on the real line that majorizes the characteristic function of the interval $[1, N]$ and such that its Fourier transform $\hat{F}_{1}$ vanishes when the argument is $\geq Q^{-2}$ in absolute value. We also assume that this function decreases fast enough at both infinities to ensure the convergence of the quantities appearing in the Poisson summation formula. We write

$$
\sum_{q \leq Q} \sum_{a}|S(a / q)|^{2}=\sum_{n \leq N} \varphi_{n} \sum_{q \leq Q} \sum_{a} \overline{\text { mod }{ }^{*} q} \overline{S(a / q)} e(n a / q)
$$


with $S(a / q)=\sum_{n} \varphi_{n} e(n a / q)$, and we apply Cauchy's inequality. This leads to

$$
\begin{aligned}
\left(\sum_{q \leq Q} \sum_{\bmod { }^{*} q}|S(a / q)|^{2}\right)^{2} & \leq \sum_{n \leq N}\left|\varphi_{n}\right|^{2} \sum_{1 \leq n \leq N}\left|\sum_{\substack{q \leq Q, a \bmod ^{*} q}} \overline{S(a / q)} e(n a / q)\right|^{2} \\
& \leq \sum_{n \leq N}\left|\varphi_{n}\right|^{2} \sum_{n \in \mathbb{Z}} F_{1}(n)\left|\sum_{\substack{q \leq Q,{ }^{*} \\
a \text { mod }{ }^{*} q}} \overline{S(a / q)} e(n a / q)\right|^{2} .
\end{aligned}
$$

We expand the last square:

$$
\begin{aligned}
& \sum_{n \leq N}\left|\varphi_{n}\right|^{2} \sum_{\substack{q \leq Q, a \\
\text { mod }{ }^{*} q a^{\prime}}} \sum_{\substack{q^{\prime} \leq Q, \\
\text { mod }{ }^{*} q^{\prime}}} \overline{S(a / q)} S\left(a^{\prime} / q^{\prime}\right) \sum_{n \in \mathbb{Z}} F_{1}(n) e\left(n\left(a / q-a^{\prime} / q^{\prime}\right)\right) \\
& \leq \sum_{n \leq N}\left|\varphi_{n}\right|^{2} \sum_{\substack{q \leq Q, a \\
\text { mod }{ }^{*} q}} \sum_{\substack{a^{\prime} \\
q^{\prime} \leq Q, \\
\text { mod }{ }^{*} q^{\prime}}} \overline{S(a / q)} S\left(a^{\prime} / q^{\prime}\right) \sum_{m \in \mathbb{Z}} \hat{F}_{1}\left(m-\left(a / q-a^{\prime} / q^{\prime}\right)\right)
\end{aligned}
$$

by Poisson summation formula. By our assumption, only $m=0$ and $a / q=$ $a^{\prime} / q^{\prime}$ survive in the inner summation. This gives us

$$
\sum_{q \leq Q} \sum_{a}|S(a / q)|^{2} \leq \hat{F}_{1}(0) \sum_{n \leq N}\left|\varphi_{n}\right|^{2} .
$$

Our problem is thus to minimize the value of $\hat{F}_{1}(0)$. Note that the function $F(u)=F_{1}\left(Q^{2} u+N / 2\right)$ verifies $\hat{F}(x)=Q^{2} \hat{F}_{1}\left(x / Q^{2}\right) e(x N / 2)$. We translate our conditions on $F$ :

(1) $\hat{F}(x)=0$ when $|x| \geq 1$;

(2) $F(u) \geq 0$ for all $u \in \mathbb{R}$;

(3) $F(u) \geq 1$ when $2|u| \leq N / Q^{2}$.

We take $F$ of the shape

$$
F(u)=\int_{-\infty}^{\infty} \int_{0}^{1} G(v) G(v-x) d v e(-x u) d x
$$

for some real-valued function $G$ with support on $[0,1]$. As a consequence, the function $x \mapsto \int_{0}^{1} G(v) G(v-x) d v$ has support on $[-1,1]$, so that

$$
\begin{aligned}
F(u) & =\int_{-1}^{1} \int_{0}^{1} G(v) G(v-x) d v e(-x u) d x \\
& =\int_{0}^{1} G(v) e(-v u) d v \int_{-1}^{1} G(v-x) e((v-x) u) d x \\
& =\left|\int_{0}^{1} G(v) e(-v u) d v\right|^{2} .
\end{aligned}
$$


This representation thus ensures us that $\hat{F}(x)=0$ when $|x| \geq 1$ and that $F(u) \geq 0$ for all $u \in \mathbb{R}$. Our problem reduces to minimizing

$$
\hat{F}(0)=\int_{0}^{1} G(v)^{2} d v=\int_{-\infty}^{\infty}|\hat{G}(u)|^{2} d u
$$

when

$$
\left|\int_{0}^{1} G(v) e(-v u) d v\right| \geq 1 \quad(|u| \leq \delta)
$$

where $\delta=N /\left(2 Q^{2}\right)$. In our case, $\delta$ is asymptotic to $3 /\left(2 \pi^{2}\right)$ which is pretty small! We want $|\hat{G}(u)|$ to be as close to the characteristic function of the interval $[-\delta, \delta]$ as is possible and this tells us that $\hat{F}(0) \geq 2 \delta$. We have not been able to solve this extremal problem, so we decided to run experiments by taking $G$ to be a polynomial. First note the formula

$$
E_{k}(u)=\int_{0}^{1} v^{k} e(-v u) d v=\frac{k ! e(-u)}{(2 i \pi u)^{k+1}}\left(e(u)-\sum_{0 \leq \ell \leq k} \frac{(2 i \pi u)^{\ell}}{\ell !}\right) .
$$

Proof: The formula is valid for $k=0$. Assume it has been proved for $k$. An integration by parts yields

$$
\begin{aligned}
\int_{0}^{1} v^{k+1} e(-v u) d v & =\frac{e(-u)}{-2 i \pi u}+\frac{(k+1)}{2 i \pi u} E_{k}(u) \\
& =\frac{e(-u)}{-2 i \pi u}+\frac{(k+1) ! e(-u)}{(2 i \pi u)^{k+2}}\left(e(u)-\sum_{0 \leq \ell \leq k} \frac{(2 i \pi u)^{\ell}}{\ell !}\right) \\
& =\frac{(k+1) ! e(-u)}{(2 i \pi u)^{k+2}}\left(e(u)-\sum_{0 \leq \ell \leq k} \frac{(2 i \pi u)^{\ell}}{\ell !}-\frac{(2 i \pi u)^{k+1}}{(k+1) !}\right)
\end{aligned}
$$

as required.

On taking simply $G_{0}(v)=1$, we already get an improvement on the usual large sieve inequality: the largest eigenvalues is less than $3.552 \cdot N$ if $Q$ is large enough, instead of $\left(1+\pi^{2} / 3\right) N \leq 4.290 \cdot N$. The choice $1+v(1-v) / 2$ yields the bound $3.548 \cdot N$. Note that its $L^{2}$-norm is $\sqrt{47 / 40}$ and not 1 ; we set $G_{1}(v)=(1+v(1-v) / 2) / \sqrt{47 / 40}$. As a matter of fact, we tried every polynomial $1+a_{1} v+a_{2} v^{2}+a_{3} v^{3}+a_{4} v^{4}$ with the $a_{i}$ 's ranging $\left\{2 \frac{k}{20}-1, k \in\{0, \ldots, 20\}\right\}$.

We explored the situation further, but on restricting our attention to polynomials of shape $1+b_{1} v(1-v)+b_{2} v^{2}(1-v)^{2}+b_{3} v^{3}(1-v)^{3}+b_{4} v^{4}(1-v)^{4}$ with the $b_{i}$ 's ranging $\left\{2 \frac{k}{20}-1, k \in\{0, \ldots, 20\}\right\}$. We found the polynomial $1+\frac{1}{2} v(1-v)+\frac{3}{10} v^{4}(1-v)^{4}$ (of $\mathrm{L}^{2}$-norm $\left.\sqrt{8577013 / 7293000}\right)$ which leads to a 
very marginal improvement: $3.547411612 \cdot N$ instead of the $3.547411644 \cdot N$ obtained with $G_{1}(v)$. We further set

$$
G_{2}(v)=\left(1+\frac{1}{2} v(1-v)+\frac{3}{10} v^{4}(1-v)^{4}\right) / \sqrt{8577013 / 7293000} .
$$

We record some more formulae in case they be of use to the reader. With $p=2 \pi u$, we have

$\frac{47}{40}\left|\hat{G}_{1}(u)\right|^{2}=\frac{4\left(1+p^{4}\right)(1-\cos p)-4 p^{3} \sin p+p^{2}(9-7 \cos p)-4 p \sin p}{2 p^{6}}$

with $\left|\hat{G}_{1}(0)\right|^{2}=845 / 846=0.998817 \cdots$. Concerning $G_{2}$, we get

$$
\begin{aligned}
& \frac{8577013}{7293000} 50 p^{18}\left|\hat{G}_{2}(u)\right|^{2} \\
& =p\left(p^{6}-288 p^{2}+12096\right)\left(5 p^{8}+5 p^{6}+36 p^{4}-6480 p^{2}+60480\right) \sin p \\
& +\left(10 p^{8}-5 p^{7}+10 p^{6}+72 p^{4}+1440 p^{3}-12960 p^{2}-60480 p+120960\right) \\
& \left(10 p^{8}+5 p^{7}+10 p^{6}+72 p^{4}-1440 p^{3}-12960 p^{2}+60480 p+120960\right) \cos p \\
& +100 p^{16}+225 p^{14}+1540 p^{12}-272160 p^{10}+2769984 p^{8}+2626560 p^{6} \\
& +11197440 p^{4}+522547200 p^{2}+14631321600
\end{aligned}
$$

and $\left|\hat{G}_{2}(0)\right|^{2}=6296503928 / 6304104555=0.998794 \cdots$.

Figure 1. Plot of the modulus square of the Fourier transform of $G_{0}, G_{1}$ and $G_{2}$. 
The plot corresponding to $G_{0}$ starts above the ones of $G_{1}$ and $G_{2}$, and dips slightly below around 0.1.the one of $G_{1}$ while the one of $G_{2}$ is indistinguishable from the one of $G_{1}$.

The above proof can easily be generalized to get the following Theorem:

Theorem 2.1. When $\mathcal{X}$ be a subset of $\mathbb{R} / \mathbb{Z}$ such that $\min \left\|x-x^{\prime}\right\| \geq \delta$ where $\|y\|$ is the distance on the unit circle. Let us assume that $N \delta \leq 9 / 10$. We have

$$
\sum_{x}\left|\sum_{n \leq N} \varphi_{n} e(n x)\right|^{2} \leq \sum_{n}\left|\varphi_{n}\right|^{2}\left(1+\left|\hat{G}_{2}(N \delta)\right|^{-2}\right) \delta^{-1}
$$

where $\left(\varphi_{n}\right)$ is any complex sequence.

In fact, when $N \delta$ is small, at least when $N \delta \leq 1 / 10$, it is better to use $G_{0}$ instead of $G_{2}$.

In particular, when $N=\Phi(Q)$ and $Q$ is large enough, we have

$$
\sum_{q \leq Q} \sum_{\bmod { }^{*} q}\left|\sum_{n \leq N} \varphi_{n} e(n a / q)\right|^{2} \leq 3.547411612 \cdot N \cdot \sum_{n}\left|\varphi_{n}\right|^{2}
$$

\section{On the moments}

Our first datas are the first $H$ (with $H=100$ ) moments. We computed them by computing the successive powers of the matrix $M$, on using 100 digits. We recomputed the first 15 moments for $Q=62$ with 150 digits to check the accuracy and got an optimal result. We further used 110 digits for $Q=66$ and $Q=70$. More precisely, we precomputed $M^{2^{k}}$ for $0 \leq k \leq 6$, as well as $M^{3}$. We then computed each $M^{h}$ for $h$ a multiple of 4 , and obtained this way the trace of $M^{h}, M^{h} \times M, M^{h} \times M^{2}$ and $M^{h} \times M^{3}$. This turned out to be a good compromise between storage problems and speed. The computation have been run GP/PARI with compiled scripts via GP2C. The scripts are available on request.

We show below a plot of

$$
h \mapsto \log \left(\operatorname{tr} M^{h} / N^{h+1}\right) .(h \geq 1)
$$

A strong regularity appears.

Let us first recall some known facts of these moments, see (3.1) below for their definition. Hausdorff determined in [4], [5] and [6] necessary and sufficient conditions for the sequence $\left(m_{h}^{h}\right)$ to be associated with a positive measure on $[0,1]$. The paper [8] contains a very clear survey of the situation. This condition comes simply from the fact that

$$
\int_{0}^{1} t^{k}(1-t)^{\ell} d D(t) \geq 0
$$


Figure 2. The moments on a logarithmic scale for $Q=40,46,50,54,58$

We expand the polynomial, replace each $\int_{0}^{1} t^{h} d D(t)$ by $m_{h}^{h}$ and get the condition that these should verify.

Seeing this regularity, it is tempting to try to modelize it; Let us first examine what to expect. On dividing by the largest eigenvalue, our asymptotical moment has the shape $\int_{0}^{1} t^{h} d D_{0}(t)$. If we assume that $D_{0}$ has a derivative, and that is what we are trying to plot!, it has the shape $\int_{0}^{1} t^{h} \delta(t) d t$.

Theorem 3.1. Assume $\delta$ is in $L^{1}([0,1])$. Assume there is a $\in[0,1[$, two positive constant $C$ and $B$, and an integer $q \geq 1$ such that

$$
\left|\delta(t)-C(1-t)^{q}\right| \leq B(1-t)^{q+1} \quad \forall t \in[a, 1] .
$$

Then

$$
\left(\int_{0}^{1} t^{h} \delta(t) d t\right)^{1 / h}=1-q \frac{\log h}{h}+\frac{\log (C q !)}{h}+\mathcal{O}\left((\log h)^{2} / h^{2}\right) .
$$

Proof: Indeed, an integration by parts yields readily

$$
\int_{0}^{1} t^{h}(1-t)^{\ell} d t=\frac{\ell ! h !}{(h+\ell+1) !}
$$


and thus

$$
\begin{aligned}
\int_{0}^{1} t^{h} \delta(t) d t= & \int_{a}^{1} t^{h} \delta(t) d t+\int_{0}^{a} t^{h} \delta(t) d t \\
= & C \int_{0}^{1} t^{h}(1-t)^{q} d t \\
& \quad+\mathcal{O}^{*}\left(B \int_{0}^{1} t^{h}(1-t)^{q+1} d t+a^{h}\left(C+B+\|\delta\|_{1}\right)\right) \\
= & \frac{C q ! h !}{(h+q+1) !}+\mathcal{O}^{*}\left(\frac{B(q+1) ! h !}{(h+q+2) !} a^{h}\left(C+B+\|\delta\|_{1}\right)\right) .
\end{aligned}
$$

We deduce from this that the moment to the power $1 / h$ equals

$$
\left(\frac{C q ! h !}{(h+q+1) !}\right)^{1 / h}\left(1+\mathcal{O}\left(\frac{B(q+1)}{h+q+2}+(h+q+1)^{q+1} a^{h}\right)\right)^{1 / h} .
$$

The logarithm of the main term is

$$
\left(\log (C q !)-q \log h-\sum_{1 \leq \ell \leq q+1} \log \left(1+\frac{\ell}{h}\right)\right) / h
$$

which is $(\log (C q !)-q \log h+\mathcal{O}(1 / h)) / h$ while the error term is simply $1+\mathcal{O}\left(1 / h^{2}\right)$. The theorem follows readily.

This theorem says in essence that having a mild regularity throughout the interval and a somewhat stronger one near to the largest point of the support of our distribution are enough to ensure a convergence of the moments in $(\log h) / h$. Having this in mind, we tried to fit the real curve with a regular one of equation $h \mapsto A+B(\log h) / h$. Let us first recall rapidly how this can be achieved via a least squares estimate. We set

$$
m_{h}(N)=\left(\sum_{1 \leq k \leq N} \lambda_{i}^{h} / N^{h+1}\right)^{1 / h} .
$$

We should minimize

$$
\begin{aligned}
\sum_{1 \leq h \leq H}\left(m_{h}(N)\right. & \left.-\sum_{i} A_{i} f_{i}(h)\right)^{2}=\sum_{1 \leq h \leq H} m_{h}(N)^{2} \\
& -2 \sum_{i} A_{i} \sum_{1 \leq h \leq H} m_{h}(N) f_{i}(h)+\sum_{1 \leq h \leq H}\left(\sum_{i} A_{i} f_{i}(h)\right)^{2}
\end{aligned}
$$

for some chosen functions $f_{i}$. In our main example, we take only the two functions $f_{1}(h)=1$ and $f_{2}(h)=\log (h) / h$. The vanishing of the partial 
derivatives in $A_{i}$ of the above quadratic form leads to a linear system:

$$
\forall i, \quad \sum_{j} A_{j} \sum_{1 \leq h \leq H} f_{i}(h) f_{j}(h)=\sum_{1 \leq h \leq H} m_{h}(N) f_{i}(h) .
$$

This is readily solved. The surprise was that the fit was not very good, even when starting the fit only for the moments of order $\geq 5$. In turn, we obtained very good results with fits of the shape

$$
A_{1}+A_{2} \frac{\log h}{h}+A_{3} \frac{\log ^{2} h}{h}+A_{4} \frac{\log ^{3} h}{h} .
$$

Theorem 3.2. For $N \in\{40,46,50,54,58,62,66,70\}$ and $h$ ranging $\{5,6, \ldots, 100\}$, we have

$$
\left|m_{h}(N)-A_{1}-A_{2} \frac{\log h}{h}-A_{3} \frac{\log ^{2} h}{h}-A_{4} \frac{\log ^{3} h}{h}\right| \leq B
$$

where the coefficients are given by the following table:

\begin{tabular}{|c||c|c|c|c||c|}
\hline$N$ & $A_{1}$ & $A_{2}$ & $A_{3}$ & $A_{4}$ & $B$ \\
\hline 40 & 2.7 & -0.788 & -2.865 & 0.674 & 0.0126 \\
\hline 46 & 2.727 & -0.823 & -2.876 & 0.661 & 0.0136 \\
\hline 50 & 2.743 & -0.848 & -2.871 & 0.648 & 0.0167 \\
\hline 54 & 2.758 & -0.839 & -2.901 & 0.646 & 0.0188 \\
\hline 58 & 2.778 & -0.878 & -2.903 & 0.639 & 0.0444 \\
\hline 62 & 2.792 & -0.917 & -2.883 & 0.625 & 0.0160 \\
\hline 66 & 2.808 & -0.932 & -2.900 & 0.623 & 0.0573 \\
\hline 70 & 2.822 & -0.952 & -2.889 & 0.620 & 0.0571 \\
\hline \hline Average $[40-70]$ & 2.766 & -0.872 & -2.889 & 0.642 & 0.0837 \\
\hline Average $[58-70]$ & 2.870 & -1.058 & -2.893 & 0.596 & 0.0340 \\
\hline
\end{tabular}

Here is a plot of the moments (to the power $1 / h$ ), which the average plot included.

The top curve corresponds to $Q=58$.

The maximum of the average fit is obtained close to $h=500$ and has value $2.833 \cdots$. As a consequence, one may ask whether it is true that

$$
\sum_{q \leq Q a} \sum_{\bmod *_{q}}\left|\sum_{n \leq N} \varphi_{n} e(n a / q)\right|^{2} \stackrel{?}{\leq} 3.2 N \sum_{n}\left|\varphi_{n}\right|^{2} \quad(N=\Phi(Q)) .
$$

The graph we produce in the last section goes in favor of this guess. The numerical datas are however too flimsy to term that a conjecture, or to even guess the optimal constant. 
Figure 3. The moments for $Q=40,46,50,54,58$ and the average fit

\section{On Jackson's polynomials}

Approximation and interpolation of the circle. We essentially follow the notations of [11] and define for any non-negative integer $n$

(4.1) $K_{n}(v)=\frac{2}{n+1}\left(\frac{\sin \frac{n+1}{2} v}{2 \sin (v / 2)}\right)^{2}=\frac{1}{2}+\frac{1}{n+1} \sum_{1 \leq \ell \leq n}(n+1-\ell) \cos \ell v$

as well as

$$
r_{k, n}=r_{0, n}+\frac{2 \pi k}{n+1}
$$

where $r_{0, n}$ is an arbitrary real number. Note that $K_{n}(0)=(n+1) / 2$, $K_{n}(v) \geq 0$ and $\int_{0}^{2 \pi} K_{n}(v) d v=1 / 2$. We define the Jackson polynomial of a $2 \pi$-periodic function $f$ to be

$$
J_{n}(f, u)=\frac{2}{n+2} \sum_{0 \leq k \leq n} f\left(r_{k, n}\right) K_{n}\left(u-r_{k, n}\right) .
$$


These polynomials are uniquely determined by the interpolation property:

$$
J\left(f, r_{k, n}\right)=f\left(r_{k, n}\right), \quad J^{\prime}\left(f, r_{k, n}\right)=0 . \quad(0 \leq k \leq n)
$$

Approximation and interpolation of the segment $[-1,1]$. We are interested in approximating a function $F$ on $[-1,1]$ by polynomials. To do so, we consider

$$
F(\cos u)=f(u)
$$

where $f$ is $2 \pi$-periodical with the additionnal property that

$$
f(2 \pi-u)=f(u) .
$$

We take $r_{0, n}=0$ so that $f\left(r_{n+1-k, n}\right)=f\left(r_{k, n}\right)$, from which we infer that

$$
J_{n}(f, u)=\frac{1}{n+2} \sum_{0 \leq k \leq n} f\left(r_{k, n}\right)\left(K_{n}\left(u-r_{k, n}\right)+K_{n}\left(u-r_{n+1-k, n}\right)\right) .
$$

We get (with $t=\cos u$ and $t^{*}=\sin u$ )

$$
\begin{aligned}
2 \cos \left(\ell u-\ell r_{k, n}\right)= & e^{i \ell u-i r_{k, n}}+e^{-i \ell u+i \ell r_{k, n}} \\
= & e^{i \ell r_{k, n}}\left(t+i t^{*}\right)^{\ell}+e^{-i \ell r_{k, n}}\left(t-i t^{*}\right)^{\ell} \\
= & \sum_{0 \leq m \leq \ell}\left(\begin{array}{c}
\ell \\
m
\end{array}\right) t^{m}\left(e^{i \ell r_{k, n}}\left(i t^{*}\right)^{(\ell-m)}+e^{-i \ell r_{k, n}}\left(-i t^{*}\right)^{(\ell-m)}\right) \\
= & \sum_{\substack{0 \leq m \leq \ell, m \equiv \ell[2]}}\left(\begin{array}{c}
\ell \\
m
\end{array}\right) t^{m}\left(i t^{*}\right)^{(\ell-m)}\left(e^{i \ell r_{k, n}}+e^{-i \ell r_{k, n}}\right) \\
& +\sum_{\substack{0 \leq m \leq \ell, m \equiv \ell+1[2]}}\left(\begin{array}{c}
\ell \\
m
\end{array}\right) t^{m}\left(i t^{*}\right)^{(\ell-m)}\left(e^{i \ell r_{k, n}}-e^{-i \ell r_{k, n}}\right) .
\end{aligned}
$$

Since $e^{i r_{n+1-k, n}}=e^{-i r_{k, n}}$, we find that

$$
\begin{aligned}
& \cos \left(\ell u-\ell r_{k, n}\right)+\cos \left(\ell u-\ell r_{n+1-k, n}\right) \\
& =\sum_{\substack{0 \leq m \leq \ell, m \equiv \ell[2]}}\left(\begin{array}{c}
\ell \\
m
\end{array}\right) t^{m}\left(i t^{*}\right)^{(\ell-m)}\left(e^{i \ell r_{k, n}}+e^{-i \ell r_{k, n}}\right)
\end{aligned}
$$

which is a polynomial in $t$, on recalling that $t^{* 2}=1-t^{2}$. Consequently, we may write

$$
\tilde{J}_{n}(F, t)=J_{n}(f, u)=\frac{1}{n+2} \sum_{0 \leq k \leq n} F\left(\cos r_{k, n}\right) \tilde{K}_{k, n}(t)
$$


for the polynomials $\tilde{K}_{k, n}(t)$ defined by

$$
\tilde{K}_{k, n}(t)=1+2 \sum_{1 \leq \ell \leq n}\left(1-\frac{\ell}{n+1}\right) \sum_{\substack{0 \leq m \leq \ell \\
m \equiv \ell[2]}}\left(\begin{array}{c}
\ell \\
m
\end{array}\right) t^{m}\left(t^{2}-1\right)^{(\ell-m) / 2} \cos \left(\ell r_{k, n}\right) .
$$

We also have

$$
\tilde{K}_{k, n}(t)=\tilde{K}_{n+1-k, n}(t)=K_{n}\left(u-r_{k, n}\right)+K_{n}\left(u-r_{n+1-k, n}\right)
$$

and as a consequence, we get $\tilde{K}_{k, n}(t) \geq 0$ and:

$$
\tilde{K}_{k, n}\left(\cos r_{k, n}\right) \geq(n+1) / 2, \quad \int_{-1}^{1} \tilde{K}_{k, n}(t) \frac{d t}{\sqrt{1-t^{2}}}=1 / 2
$$

Indeed, set $t=\cos \left(u-r_{k, n}\right)$, so that $d t=-\sin \left(u-r_{k, n}\right) d u=-\sqrt{1-t^{2}} d u$ on the first part and $t=\cos \left(u-r_{n+1-k, n}\right)$ on the second one. Furthermore, we have

$$
\tilde{J}_{n}\left(F, \cos r_{k, n}\right)=F\left(\cos r_{k, n}\right), \quad \tilde{J}_{n}^{\prime}\left(F, \cos r_{k, n}\right)=0 . \quad(0 \leq k \leq n)
$$

Since we want to approximate characteristic functions of intervals, these conditions are of course exactly what we need!

\section{Plotting and interpolation}

We used a Bernstein interpolation of degree 2 between two of our (guessed!) points, and here is how we proceeded; given two consecutive points $B$ and $C$, we looked at the previous point $A$ and the next one $D$. When the lines joining $A$ and $B$ and $C$ and $D$ had an intersection with an abscissa between the one of $B$ and the one of $C$ (practically: everywhere but at the beginning of the plot), we computed this intersection, say $I$ and plotted the arc

$$
(1-t)^{2} B+2 t(1-t) I+t^{2} C, \quad t \in[0,1]
$$

which lies in the convex hull of $B, I$ and $C$.

Let us record some (trivial) details concerning the computation of the intersection of $\lambda A+(1-\lambda) B$ with $\mu C+(1-\mu) D$. They are however necessary to write the plotting script! We are to determine $\lambda$ and $\mu$ so that

$$
\lambda x_{A}+(1-\lambda) x_{B}=\mu x_{C}+(1-\mu) x_{D}
$$

hence, when $x_{A} \neq x_{B}$, we have $\lambda=\left(\mu\left(x_{C}-x_{D}\right)+x_{D}-x_{B}\right) /\left(x_{A}-x_{B}\right)$ and similarly with $y$ 's intead of $x$ 's. After some manipulations, we reach

$$
\begin{aligned}
\mu\left(\left(x_{C}-x_{D}\right)\left(y_{A}-y_{B}\right)-\right. & \left.\left(x_{A}-x_{B}\right)\left(y_{C}-y_{D}\right)\right) \\
& =\left(y_{D}-y_{B}\right)\left(x_{A}-x_{B}\right)-\left(x_{D}-x_{B}\right)\left(y_{A}-y_{B}\right) .
\end{aligned}
$$

This can also be written as $\mu \operatorname{det}(\overrightarrow{D C}, \overrightarrow{B A})=\operatorname{det}(\overrightarrow{A B}, \overrightarrow{B D})$. The determinant vanishes when the two lines are parallel. 
Figure 4. Estimated density of eigenvalues $/ N$ for $Q=$ $58,62,66$ and 70

A shape indeed emerges from these drawings, but the convergence appears to be very slow. The problem concerning what these drawings can represent is well treated in [2]. The authors show there that a sequence of measures, say $\left(\mu_{n}\right)_{n \geq 1}$, each of them satisfying

$$
\forall k \leq n, \quad \int t^{k} d \mu_{n}=\int t^{k} d \mu,
$$

converges weakly towards $\mu$. However, they also exhibit a sequence of functions (and a measure $\mu$ ) such that

$$
\forall k \leq n, \quad \int t^{k} f_{n}(t) d \mu=\int t^{k} f(t) d \mu
$$

while $\left(f_{n}\right)_{n \geq 1}$ does not converge towards $f$. This is most probably a pathological case, but it is worth mentionning it, especially in the light of section 3.

\section{References}

[1] E. Bombieri. Le grand crible dans la théorie analytique des nombres. Astérisque, 18:103pp, 1987. 
[2] J.M. Borwein and A.S. Lewis. On the convergence of moment problems. Trans. Amer. Math. Soc., 325(1):249-271, 1991.

[3] G. Greaves. An algorithm for the Hausdorff moment problem. Numerische Mathematik, 39(2):231-238, 1982.

[4] F. Hausdorff. Summationsmethoden und Momentfolgen. I. Math. Z., 9:74-109, 1921.

[5] F. Hausdorff. Summationsmethoden und Momentfolgen. II. Math. Z., 9:280-299, 1921.

[6] F. Hausdorff. Momentenprobleme für ein endliches Intervall. Math. Z., 16:220-248, 1923.

[7] H.L. Montgomery. Topics in Multiplicative Number Theory. Lecture Notes in Mathematics (Berlin), 227:178pp, 1971.

[8] I.J. Schoenberg R. Askey and A. Sharma. Hausdorff's moment problem and expansions in Legendre polynomials. J. Math. Anal. Appl., 86:237-245, 1982.

[9] O. Ramaré. Eigenvalues in the large sieve inequality. Funct. Approximatio, Comment. Math., 37:7-35, 2007.

[10] A. Selberg. Collected papers. Springer-Verlag, II:251pp, 1991.

[11] J. Szabados. On the convergence and saturation problem of the Jackson polynomials. Acta Math. Acad. Sci. Hungar., 24:399-406, 1973

Olivier RAMARÉ

Laboratoire CNRS Paul Painlevé,

Université Lille 1,

59655 Villeneuve d'Ascq cedex, France

E-mail : ramare@math.univ-lille1.fr 